\title{
ARQUITETURA DA ÁRVORE BRÔNQUICA NO SAGUI-DE-TUFO-PRETO: UM MODELO ANIMAL EXPERIMENTAL PARA LESÕES DO SISTEMA RESPIRATÓRIO
}

\author{
Luana Célia Stunitz da Silva1, Thaís Borges Lessa1, Paulo Ramos da Silva Santos", \\ Rose Eli Grassi Rici', Pedro Primo Bombonato1, Carlos Eduardo Ambrósio² \\ 1 FMVZ - USP \\ 2 FZEA - USP \\ Correspondência: Luana Silva: luanastunitz@usp.br
}

\begin{abstract}
RESUMO: O sagui-de-tufo-preto (Callithrix penicillata), bem como outros representantes do gênero Callithrix, possui uma grande importância na área da pesquisa, principalmente em estudos biomédicos. Entretanto existe ainda uma carência em pesquisas básicas, tais como aquelas que abarcam aspectos de sua anatomia e de sua fisiologia. Considerando a escassez de informações acerca da morfologia do sistema respiratório em saguis da espécie Callithrix penicillata, objetivou-se neste estudo descrever os aspectos histológicos e ultraestruturais da traquéia, dos brônquios e do pulmão destes animais por meio do uso da microscopia eletrônica de varredura (MEV) e da microscopia de luz. Para a avaliação foram utilizados cinco espécimes adultos, 3 fêmeas e 2 machos, que vieram a óbito por causas naturais. Na MEV da traquéia e brônquio observaram-se uma superfície densa de capilares salientes, a cartilagem hialina em formato de "C" e o feixe de músculo liso disposto em formato helicoidal. Já no tocante aos pulmões houve a observação das mesmas estruturas típicas de um pulmão de mamífero. Na microscopia de luz tanto para a traquéia, brônquios e pulmões as estruturas foram similares às já descritas para demais espécies de primatas não-humanos. Os dados obtidos nesta pesquisa elucidam a morfologia histológica e ultraestrutural da traquéia, brônquios e pulmões do sagui-de-tufo-preto (Callithrix penicillata). Servindo assim de subsídio para estudos de anatomia comparada bem como para o conhecimento acerca da morfologia de tal espécie de primata não-humano.
\end{abstract}

Palavras-chave: anatomia veterinária; microscopia de luz; microscopia eletrônica de varredura; saguis; respiração

\section{ARCHITECTURE OF THE BRONCHIAL TREE OF BLACK-TUFTED-EAR- MARMOSET: AN EXPERIMENTAL ANIMAL MODEL FOR RESPIRATORY SYSTEM INJURIES}

\begin{abstract}
The black-tufted-ear-marmoset (Callithrix penicillata) as well as other representatives of the genus Callithrix, has a great importance in research, especially in biomedical studies. However there is still a lack in basic research, such as those covering aspects of their anatomy and physiology. Given the scarcity of information about the morphology of the respiratory system in Callithrix penicillata, this study aimed to describe the histological and ultrastructural aspects of the trachea, bronchus and lung of these animals through the use of scanning electron microscopy (SEM) and light microscopy. For the evaluation we used five adult specimens, three females and two males, which they had died of natural causes. SEM in the trachea and bronchi were observed a dense surface of capillaries protruding numerous pores of Kohn, the hyaline cartilage and smooth muscle bundle arranged in a spiral. Already with regard to the lungs was the observation of the same structures typical of a mammalian lung. In light microscopy both the trachea, bronchi and lungs were similar to the structures already described for other species of primates non-human. The data obtained in this study elucidate the histological and ultrastructural morphology of the trachea, bronchi and lungs of the black-tufted-ear-marmoset (Callithrix penicillata). Thus serving as a grant for studies of comparative anatomy as well as knowledge about the morphology of this kind of non-human primate. Key Words: : light microscopy; marmosets; respiration; scanning electron microscopy; veterinary anatomy
\end{abstract}




\section{INTRODUÇÃO}

O Callithrix penicillata, assim como outros saguis do gênero Callithrix, possui uma pelagem estriada no dorso e na cauda, apresentando ornamentos pilosos nas orelhas. A presença de tufos pré-auriculares longos e negros em forma de pincel assegura-lhe o nome de sagui-de-tufo-preto, e os diferenciam de outras espécies do gênero. É também conhecido como sagui-do-cerrado ou mico-estrela (Auricchio, 1995; BiccaMarques et al., 2006).

Este primata habita as florestas e matas de galeria do cerrado brasileiro apresentando uma ampla distribuição na natureza, pois abrange os estados do Maranhão e sudeste do Piauí até o norte de São Paulo (Rylands et al., 1997). Vive cerca de 10 anos em vida livre, 16 anos em cativeiro e atinge a maturidade sexual aos 18 meses. É considerado um animal de pequeno porte, por abarcar um valor de 300 a $450 \mathrm{~g}$, sendo considerado insetívorogumívoro, por sua alimentação se basear em frutos, insetos, flores, néctar e exsudatos de plantas (Auricchio, 1995; Bicca-Marques et al., 2006).

Tais animais, bem como outros representantes do gênero Callithrix, possuem uma grande importância na área da pesquisa, principalmente em estudos biomédicos, não somente por sua ocorrência nos ecossistemas da América do Sul, mas também devido ao seu pequeno porte, facilidade no manejo, biossegurança e peculiaridades fisiológicas (Abbott et al., 2003; Mansfield, 2003).

No entanto apesar do aumento da demanda desses pequenos primatas existe ainda uma carência em pesquisas básicas, tais como aquelas que abrangem aspectos de sua morfologia e de sua fisiologia. Como, por exemplo, as que subsidiam questões que envolvem as características do sistema respiratório. $\mathrm{E}$ sabe-se que $\mathrm{o}$ conhecimento acerca da anatomia e a designação adequada das estruturas corpóreas são de suma importância para o aprendizado, o desenvolvimento e o avanço de todas as áreas da ciência médica (Schaeffter, 1996).

A função primária do sistema respiratório é a de possibilitar a entrada de oxigênio e a eliminação de dióxido de carbono do corpo. E tal sistema pode ser dividido, de forma estrutural, em sistema respiratório superior, o qual inclui o nariz, cavidades nasais, seios paranasais e nasofaringe, e o sistema respiratório inferior, este englobando a laringe, traquéia, brônquios e pulmões. De acordo com Dyce et al. (2010) a traquéia em conjunto com os brônquios formam um sistema contínuo de tubos que conduzem o ar entre a laringe e os bronquíolos, estes últimos presentes nos pulmões, e, por apresentarem estruturas muito similares entre si, são denominados, por alguns autores, de árvore traqueobronquial. Além disso, no aspecto funcional o sistema respiratório como um todo pode ser dividido em porção condutora e porção respiratória (Liebich e König, 2005; Samuelson et al., 2007; Akers e Denbow, 2008).

$E$ nesse contexto sabe-se que a estrutura e a forma do sistema respiratório inferior, que varia entre os indivíduos e entre as espécies, refletem a demanda metabólica e de oxigênio, estas as quais podem ser atribuídas ao hábitat de cada animal (Miller et al., 1993; lbe et al., 2011). Diretamente relacionado aos animais do gênero Callithrix, estes podem ser modelos animais úteis para $o$ estudo da capacidade adaptativa do sistema respiratório, em relação a diferentes demandas energéticas, bem como também nas investigações da influência de fatores ambientais adversos, tais como a poluição do ar nas estruturas pulmonares (Barbier e Bachofen, 2000).

No tocante especificamente aos pulmões, diversas foram as pesquisas já 
realizadas para com estas estruturas em primatas não-humanos. $O$ que é justificável tendo em vista a proximidade filogenética com os seres humanos, e assim se tornam, quando comparados a outros mamíferos, melhores modelos para estudo da estrutura, função e patologia pulmonar (Maina, 1987).

Mediante esses informes e considerando a escassez de informações acerca da morfologia histológica e ultraestrutural do sistema respiratório em saguis da espécie Callithrix penicillata, objetivou-se neste estudo estabelecer a morfologia da traquéia, brônquios e pulmões de tais animais por meio do uso da microscopia eletrônica de varredura e da microscopia de luz.

\section{MATERIAL E MÉTODOS}

Para a avaliação foram utilizados cinco espécimes adultos de Callithrix penicillata (sagui-de-tufo-preto) sendo 3 fêmeas e 2 machos, os quais vieram a óbito por causas naturais. Os animais eram provenientes de um criadouro comercial de animais selvagens localizado na cidade de Atibaia-SP (Brasil), e foram doados para o Laboratório de Anatomia dos Animais Domésticos e Silvestres da Faculdade de Medicina Veterinária e Zootecnia da Universidade de São Paulo (FMVZUSP) para estudos anatômicos. Após seu recebimento os animais foram fixados por imersão em solução de formaldeído $10 \%$ por um tempo mínimo de 72 horas para a sua posterior dissecação.

Após a fixação procedeu-se com uma incisão e rebatimento em sentido dorsal da pele na linha mediana ventral, do processo xifóide do osso esterno até o manúbrio, com posterior retirada da fáscia subcutânea, grupos musculares e costelas em antímeros. Permitindo assim 0 acesso a algumas das estruturas do sistema respiratório inferior, sendo estas a traquéia torácica, os brônquios e os pulmões. Em seguida procedeu-se com a remoção deste conjunto como um todo para a retirada de amostras para o processamento à microscopia eletrônica de varredura e microscopia de luz, ambos realizados na FMVZ-USP.

$\mathrm{Na}$ microscopia eletrônica de varredura as amostras inicialmente foram lavadas em água destilada por 20 minutos e pós-fixadas em solução de tetróxido de Ósmio a 1\% (Polyscience ${ }^{\circledR}$, Inc. USA) por 2 horas. Em seguida 0 material foi desidratado em séries crescentes de alcoóis (50\% a 100\%), durante 30 minutos em cada série, com posterior secagem em aparelho de ponto crítico Balzers CPD 020, utilizando-se de $\mathrm{CO}_{2}$ líquido. A seguir tais amostras foram montadas em bases metálicas de alumínio ("stub") apropriadas para a microscopia eletrônica de varredura, por meio do uso de cola de carbono. $\mathrm{Na}$ sequência, foram submetidas a um revestimento metálico ("sputting") com ouro no aparelho metalizador EMITECH K550 e analisadas e fotografadas ao microscópio eletrônico de varredura (Morgagni 268D - Phillips ${ }^{\circledR}$ ).

Para a microscopia de luz procedeu-se inicialmente com a desidratação das amostras em séries crescentes de etanol (70 a 100\%), diafanização em xilol e inclusão em Paraplast® (Leica/Germany). Permitindo assim a confecção de blocos retangulares com base de $3 \times 4 \mathrm{~cm}$ nos quais foram efetuados cortes de $5 \mu \mathrm{m}$ em micrótomo (Leica RM 2065) para a obtenção de lâminas histológicas. Estas foram então desparafinizadas em estufa a $60^{\circ} \mathrm{C}$ por 2 horas e coradas com Tricrômio de Masson e com Hematoxilina-Eosina (HE). As lâminas foram fotomicrografadas em Microscópio Olympus BX 60 acoplado a câmera Axio CAM HRc, utilizando-se o software Zeiss ${ }^{\circledR}$ KS 400 . 

para lesões do sistema respiratório

\section{RESULTADOS E DISCUSSÃO}

Em relação às variações de diâmetro encontradas nas amostras analisadas dos brônquios dos saguisde-tufo-preto, estas são peculiaridades de ocorrência comum, tanto na forma regional quanto entre as diferentes espécies animais. Sendo que tal diferença de diâmetro torna-se mais significativa quando se observa os brônquios e os bronquíolos, do que as que são constatadas na traquéia (Dyce et al., 2010).

Histologicamente

as

características dos brônquios principais, ou também denominados de brônquios primários, se assemelharam à traquéia dos animais analisados, diferindo apenas em seu menor diâmetro e na existência de placas irregulares de cartilagem. Bem como também foi observado para com os brônquios lobares, ou secundários ou intrapulmonares, os quais também apresentaram características similares à traquéia com suas devidas proporções em menor espessura e diâmetro. Desta forma houve então nestas estruturas o predomínio de uma mucosa de tecido epitelial pseudo-estratificado cilíndrico ciliado, uma lâmina própria de tecido conjuntivo frouxo rico em fibras elásticas e glândulas seromucosas, uma submucosa, uma lâmina fibrocartilaginosa com numerosos anéis de cartilagem hialina incompletos dorsalmente em formato de " $C$ " os quais estavam unidos por feixes de músculo liso que se fixavam na parte interna da cartilagem denominada de pericôndrio, e a adventícia externamente, corroborando assim com a literatura especializada (Figura 1A). E quando ocorria a transição dos brônquios para os bronquíolos, teve-se a perda dos anéis de fibrocartilagem (Junqueira e Carneiro, 2004; Liebich e König, 2005; Samuelson,2007; Akers e Denbow, 2008).
Com relação aos aspectos ultraestruturais tanto a traquéia quantos os brônquios apresentaram uma superfície densa de capilares salientes, cartilagem hialina em forma de "C" e feixe de músculo liso disposto em um aspecto helicoidal externamente (Figura $2 \mathrm{~A}$ e $2 \mathrm{~B}$ ).

As diferenças na arquitetura pulmonar entre as mais distintas espécies animais têm sido correlacionadas afetando a capacidade difusora do oxigênio, como um índice de condutância do fluxo de oxigênio dos alvéolos para dentro dos eritrócitos nos capilares pulmonares. Sendo que esta variabilidade é tratada como um parâmetro para diferenciar entre 0 normal e o patológico. Entretanto algumas estruturas são invariáveis, apesar de suas dimensões e arranjos serem influenciados por fatores funcionais dentro da relação estruturafunção do pulmão (Barbier e Bachofen, 2000).

Segundo Gehr et al. (1978) e Barbier e Bachofen (2000) diversas variações individuais dos elementos estruturais do parênquima pulmonar são passíveis de ocorrer nos seres humanos, fato este também observado quando da análise dos pulmões dos saguis-de-tufo-preto.

Análises realizadas nos pulmões do rato, camundongo e babuíno demonstraram que pulmões maiores são resultantes do aumento tanto do tamanho quanto do número das unidades ventilatórias, sendo esta última a mais prevalente. A evidência para esse fato se dá quando nota-se que o tamanho da unidade ventilatória aumenta apenas de 5 a 10 vezes do camundongo para o babuíno, embora a massa corpórea entre tais aumente mais do que três ordens de magnitude (Miller et al., 1993).

As observações do presente estudo vão de encontro com a afirmação de Barbier e Bachofen (2000) 
ao estudarem outra espécie de sagui, o Callitrhix jacchus, em que se relatou que a arquitetura e a fina estrutura do aparato pulmonar de tal espécie eram comparáveis com a de outras espécies de mamíferos, incluindo também 0 homem. A organização ultraestrutural do pulmão observado para os saguis deste estudo foi similar ao observado para demais espécies de primatas, como, por exemplo, o babuíno (Maina, 1987). Possuindo estruturas tais como vasos sanguíneos, bronquíolos os quais davam origem aos bronquíolos terminais, estes desembocando em estruturas tubulares denominadas de ductos alveolares, os quais por sua vez terminavam em "cachos" de alvéolos, os chamados sacos alveolares (Figura 2C e 2D).

Com relação ao parênquima pulmonar dos animais analisados observou-se que histologicamente 0 mesmo apresentava-se como um típico pulmão de mamífero, ou seja, era essencialmente constituído de bronquíolos, ductos alveolares, sacos alveolares, alvéolos, capilares sanguíneos circundados por musculatura lisa, septo interalveolar composto por finas fibras elásticas e de colágeno, e numerosos poros de Kohn os quais são poros interalveolares que tem por objetivo a passagem de ar e de macrófagos alveolares de um alvéolo a outro, corroborando assim com as afirmações de Junqueira e Carneiro (2004), Plopper e Adams (2006) e Samuelson (2007), e validando desta forma os achados ultraestruturais para tal primata (Figura 1B).

Nesse mesmo contexto Maina (1987) relata que o pulmão humano é estruturalmente menos adaptado para as trocas gasosas quando comparado aos dos primatas não-humanos. De acordo com Conradi et al. (1971) o pulmão da espécie Macaca irus parece ser morfometricamente melhor adaptado para as trocas gasosas do que 0 babuíno. Já para os macacos Rhesus, por serem menores e mais ativos do que os babuínos, detêm uma maior taxa metabólica de repouso a qual consequentemente representa uma melhor adaptação na troca gasosa pulmonar.

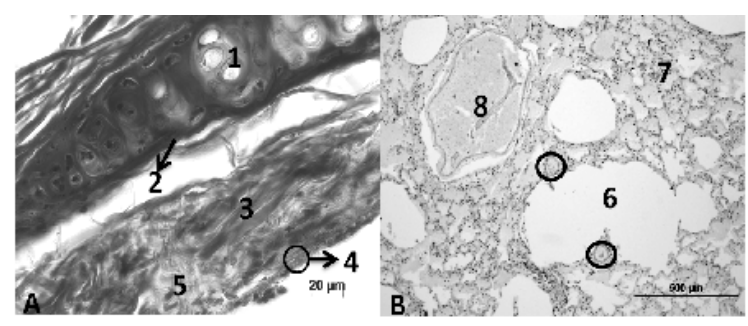

Figura 1 - Fotomicrografia de secção transversal do brônquio principal (A) e parênquima pulmonar (B) de sagui-de-tufo-preto (Callithrix penicillata). Em (A) observa-se a presença de cartilagem hialina (1), pericôndrio (2), lâmina própria de tecido conjuntivo frouxo, rico em fibras elásticas (3), mucosa com epitélio pseudo-estratificado ciliado (4) e as glândulas seromucosas (5) (Barra $=20 \mu \mathrm{m}$, Tricrômio de Masson). $\mathrm{Em}$ (B) observa-se os bronquíolos terminais (6), os alvéolos (7), vênula (8) $\mathrm{HE}$.

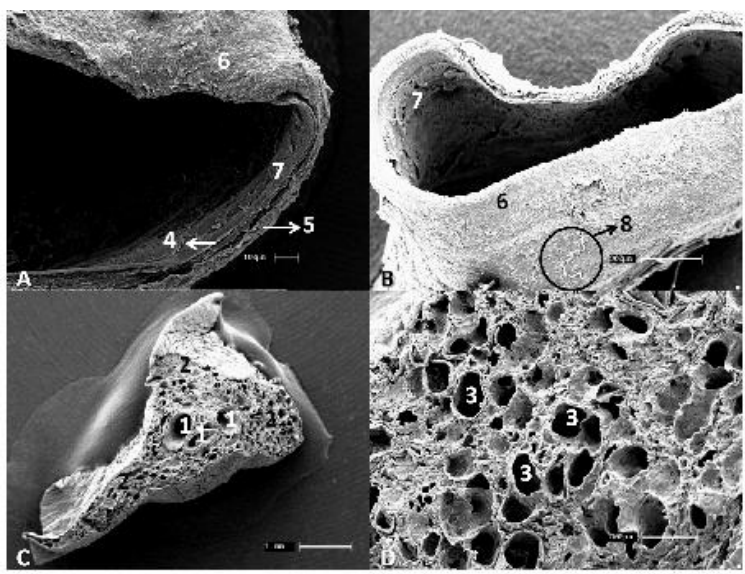

Figura 2 - Microscopia eletrônica de varredura de secção transversal da traquéia brônquios e pulmões de sagui-de-tufo-preto (Calithrix penicillata). Observa-se em (C) pulmão com os brônquios terminais (1) e parenquima pulmonar com densa rede de alveolos (2) (Barra= $10 \mu \mathrm{m})$ em (D) ductos alveolares circundados por diversos alvéolos (3) (Barra= $300 \mu \mathrm{m}) ;($ A ) Traquéia com a presença da cartilagem hialina em formato de ${ }^{2} C^{\prime}$ (4), um revestimento de musculo liso (5), tecido conjuntivo froux na submucosa e a adventicia mais extemamente (6) e tecido pseudoestratificado ciliado na mucosa (7) (Barra $=100 \mu \mathrm{m}$ ); (B) Brônqui principal ou primário com a presença de tecido conjuntivo frouxo $\mathrm{na}$ submucosa e a adventícia externamente (6), tecido pseudo-estratificado ciliado na mucosa (7) e musculatura lisa com feixes dispostos em espira (8) $($ Barra $=300 \mu \mathrm{m})$

Sendo este também o mesmo caso observado e relatado para os saguis-de-tufo-preto

(Callithrix penicillata), pois quando os mesmos são comparados com outras espécies de mamíferos de mesmo tamanho corporal, como algumas espécies de roedores, por exemplo, tais primatas possuem maiores pulmões e parecem apresentar uma maior capacidade de trocas 

para lesões do sistema respiratório

gasosas. E aliado a isso, tendo em vista que os mesmos apresentam altas demandas energéticas devido às suas atividades atléticas, apresentam de fato uma variação adaptativa pulmonar (Karas, 1987).

\section{CONCLUSÃO}

Os dados obtidos nesta pesquisa elucidam a morfologia histológica e ultraestrutural da traquéia, brônquios e pulmões do sagui-de-tufo-preto (Callithrix penicillata). Servindo assim de subsídio para estudos de anatomia comparada bem como para o conhecimento acerca da morfologia de tal espécie de primata não-humano. Pode-se concluir ainda que a partir dos resultados obtidos ocorra uma similaridade das estruturas analisadas para com as de outras espécies de mamíferos.

\section{AGRADECIMENTOS}

Ao criadouro comercial de animais selvagens, AJBSoares, pela disponibilidade e fornecimento dos animais analisados e à Médica Veterinária Marta Brito Guimarães.

\section{REFERÊNCIAS}

ABBOTT, D.H.; BARNETT, D.K.; COLMAN, R.J. et al. Aspects of Common Marmoset Basic Biology and Life history Important for Biomedical Research. Comparative Medicine, v.53, n.4, p.339-350, 2003.

AURICCHIO, P. Primatas do Brasil. São Paulo: Terra Brasilis, 1995. 168p.

AKERS, R.M.; DENBOW, D.M. Anatomy \& Physiology of Domestic Animals. 1. Ed. lowa: Blackwell Publishing, 2008. 612p.

BARBIER, A.; BACHOFEN, H. [2000]. The lung of the marmoset (Callithrix jacchus): ultrastructure and morphometric data.

Respiration Physiology, v.120, n.1, p.167-177, 2000.
BICCA-MARQUES, J.C.; SILVA, V.M.; GOMES, D.F. Ordem Primates. In: REIS, N.R.; PERACCHI, A.L.; PEDRO, W.A.; LIMA, I.P.

Mamíferos do Brasil. 1. ed. Londrina:

Universidade Estadual de Londrina, 2006, Cap. 5, p.101-148.

CONRADI, C.; BURRI, P.H.; KAPANCI, Y. et al. Lung changes after beryllium inhalation: ultrastructural and morphometric study.

Archives of Environmental Health, v.23, n.5, p.348-358, 1971.

DYCE, K.M.; SACK, W.O.; WENSING, C.J.G Textbook of Veterinary Anatomy. 4. ed. Philadelphia: W.B. Saunders Elsevier, 2010. 834p.

GEHR, P.; BACHOFEN, M.; WEIBEL, E.R. [1978]. The normal human lung: Ultrastructure and morphometric estimation of diffusion capacity. Respiration Physiology, v.32, n.2, p.121-140, 1978

IBE, C.S.; SALAMI, S.O.; ONYEANUSI, B.I. Macroscopic Anatomy of the Lower Respiratory System in a Nocturnal Burrowing Rodent: African Giant Pouched Rat (Cricetomys gambianus, Waterhouse 1840). Anatomia, Histologia, Embryologia, v.40, n.2, p.112-119, 2011.

JUNQUEIRA, L.C.; CARNEIRO, J. Histologia Básica. 10. ed. Rio de Janeiro: Guanabara Koogan, 2004. 487p.

KARAS, R.H.; TAYLOR, C.R.; JONES, J.H. et al. [1987]. Adaptive variation in the mammalian respiratory system in relation to energetic demand: VII. Flow of oxygen across the pulmonary gas exchanger. Respiration Physiology, v.69, n.1, p.101-115, 1987.

KERR, J.B. Atlas de histologia funcional. 1. Ed. São Paulo: Artes Médicas Ltda, 2000. 402 p.

LIEBICH, H.G.; KÖNIG, H.E. Introducción. In: Anatomía de los Animales Domésticos Aparato Locomotor. 2. ed. Madrid: Editorial Medica Panamericana, 2005, Cap. 1, p. 90-98.

MAINA, J.N. [1987]. The morphology and morphometry of the adult normal baboon lung (Papio anubis). Journal of Anatomy, v.150, p. 229-245, 1987.

MANSFIELD, K. [2003]. Marmoset models commonly used in biomedical research. Comparative Medicine, v.3, n.4, p.383-392, 2003. 
MILLER, F.J.; MERCER, R.R.; CRAPO, J.D.

[1993]. Lower Respiratory Tract Structure of

Laboratory Animals and Humans: Dosimetry

Implications. Aerosol Science and

Technology, v.18, n.3, p.257-271, 1993.

PLOPPER, C.G.; ADAMS, D.R. In: EURELL, J.A.;

FRAPPIER, B.L Respiratory System. Textbook of

Veterinary Histology. 6 ed. UK: Blackwell Publishing,

2006, Cap.9 p.153-169.

RYLANDS, A.B.; MITTERMEIER, R.A.;

RODRÍGUEZ-LUNA, E. [1997]. Conservation of

Neotropical Primates: Threatened Species and

an analysis of primate diversity by country an

region. Folia Primatologica, v.68, n.3-5, p134-

160, 1997

SAMUELSON, D.A. Sistema Respiratório. In:

Tratado de Histologia Veterinária. 1. ed.

Philadelphia: W.B. Saunders Elsevier, 2007,

Cap.11, p. 217-240.

\section{SCHAEFFTER, C.O.D. Aspectos ultra-}

sonográficos de rins de macaco-prego

(Cebus sp.). 1996. São Paulo, 61f. Dissertação

(Mestrado em Anatomia dos Animais

Domésticos) - Faculdade de Medicina

Veterinária e Zootecnia, Universidade de São

Paulo, 1996. 